\title{
A New Approach for MR Brain Image Segmentation using Intuitionistic Fuzzy Complement
}

\author{
P. Dhanalakshmi, $\mathrm{PhD}$ \\ Department of Mathematics, \\ Tiruppur Kumaran College For Women, \\ Tirupur,Tamil Nadu
}

\author{
M. Kavitha \\ Department of Mathematics, \\ Tiruppur Kumaran College For Women, \\ Tirupur,Tamil Nadu
}

\begin{abstract}
As medical images contain uncertainities there are difficulties in classification of images into homogeneous regions.In order to carry out this task, intuitive ways have been found out to interpret and describe the inherent ambiguity and vagueness in the medical images interms of intuitionistic fuzzy set theory.The comparison of Intuitionistic Fuzzy C-Means algorithm with three different type of Intuitionistic Fuzzy Generator(IFG) is presented in this paper.
\end{abstract}

\section{Keywords}

FCM,IFCM,Image segmentation,Intuitionistic fuzzy complement.

\section{INTRODUCTION}

Motivated from Zadeh's definition of fuzzy sets theory[9],where uncertainty or vagueness is considered only in the form of membership function.Higher order fuzzy sets have been presented by various researchers,among them Intuitionistic fuzzy sets proposed by Atanassov[1] has been a suitable tool for modelling the hesitancy arising from imprecise or/and imperfect information. This hesitation is due to the lack of knowledge or the personal error in defining the membership function.Intuitionistic fuzzy sets are defined by two characteristic functions, namely the membership and the non membership,describing the belongingness and non belongingness of an element respectively.Image segmentation[3] is one of the major step in medical image processing.Imaging can be used to visualize different anatomical parts of human body from X-ray,ultrasound,MRI etc.Brain imaging is useful in detection of brain tumor,stroke,paralysis etc.Segmentation methods that includes the classification of tissues in medical imagery can be performed using a variety of techniques.Many clustering strategies have been used such as the crisp clustering scheme and the fuzzy clustering scheme[6].The fuzzy set theory[6] which involves the idea of partial membership described by a membership function,fuzzy clustering as a soft segmentation method has been widely studied and successfully applied to image segmentation.Although the conventional FCM algorithm has a serious limitation.Chaira[4] introduced intuitionistic fuzzy entropy in objective function of conventional clustering algorithm and applied to medical images.Deepali Aneja and Tarun Kumar Rawat[5]used Yager's complement [6]to calculate hesitation factor in IFCM algorithm for the effective medical image segmentation.

In this paper,the complement defined by Klir and Bo Yuan [6]is adapted in IFCM algorithm for efficient MR brain image segmentation and compared the result with existing methods.

\section{REVIEW OF ALGORITHMS}

The image is converted into a data set of pixels which is denoted by $\mathrm{Y}$, where $\mathrm{Y}=\left\{\mathrm{y}_{1}, \mathrm{y}_{2}, \ldots, \mathrm{y}_{\mathrm{n}}\right\}$. This specifies that an image with pixels in $\mathrm{N}$-dimensional space has to be partitioned into ' $c$ ' clusters. These algorithms are based on the distance $\left(\mathrm{d}_{\mathrm{ik}}\right)$ between the centroid of the cluster $\left(v_{i}\right)$ and the particular $\operatorname{pixel}\left(\mathrm{y}_{\mathrm{k}}\right)$.

\subsection{Fuzzy C-means Algorithm}

FCM[2] is the standard fuzzy clustering algorithm.The assumption made in this technique is the prior knowledge of the no.of clusters ' $c$ '. The distance $\mathrm{d}_{\mathrm{ik}}=\left\|\mathrm{y}_{\mathrm{k}}-U_{\mathrm{i}}\right\|$ represents how far is pixel $y_{\mathrm{k}}$ from the cluster center $v_{i}$. The membership of pixel ' $y_{k}$ ' in the $i^{\text {th }}$ cluster is represented as $u_{i k}$ which satisfies,

$$
\sum_{i=1}^{c} u_{i k}=1 ; k=1,2, \ldots, n
$$

The membership function gives the probability that a pixel belongs to a specific cluster.The parameter ' $q$ ' is a weight that determinig the degree to which partial members of a cluster affect the clustering result.

The membership function and cluster centers are calculated using,

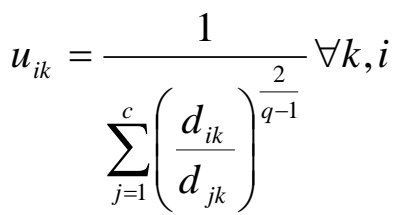

Where $1 \leq \mathrm{i} \leq \mathrm{c} ; 1 \leq \mathrm{k} \leq \mathrm{n}$ and,

$$
v_{i}=\frac{\sum_{k=1}^{n}\left(u_{i k}^{q} y_{k}\right)}{\sum_{k=1}^{n} u_{i k}^{q}} \forall i
$$

The FCM minimize the following objective function,

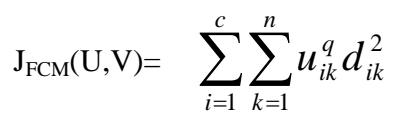

Generally used feature in image clustering is the gray level value or the intensity of the pixel.The assignment of the membership values depends on the distance between the pixel point and the cluster centroid.To minimize the cost function for FCM,low membership values are assigned when the point is far from the centroid and vice versa.The FCM algorithm is as follows: 
Step 1:Fix $\mathrm{q}>1$ and $2 \leq \mathrm{c} \leq \mathrm{n}-1$ and give $\mathrm{c}$ initial cluster centers $v_{i}$

\section{REPEAT}

Step 2:Compute $\mathrm{u}_{\mathrm{ik}}$ with $v_{i}$ by (2).

Step 3:Compute the objective function $\mathrm{J}_{\mathrm{FCM}}$ by (4)

Step 4:Update $v_{i}$ with $\mathrm{u}_{\mathrm{ik}}$ by (3).

UNTIL

$\operatorname{Max}\left|\mathrm{U}^{\text {new }}-\mathrm{U}^{\text {prev }}\right| \leq \beta$ where $\beta$ is user defined value,is satisfied.

\subsection{Intuitionistic Fuzzy C- Means \\ Algorithm}

In case of digital images, it can't be accurately defined which pixel belongs to exactly which cluster.There is some kind of hesitation related to the definition of the membership function.This idea lead to the idea of the higher fuzzy set by Atanassov in 1983[1] called as intuitionistic fuzzy set.

IFCM[4] objective function is derived from two basic terms: (i)Conventional FCM objective function which is modified by IFS(ii)IFE which shows entropy of intuitionistic fuzzy.Minimizing objective function using IFCM is:

$$
J_{I F C M}=\sum_{i=1}^{c} \sum_{k=1}^{n} u_{i k}^{q} d_{i k}^{2}+\sum_{i=1}^{c} \pi_{i}^{*} e^{1-\pi_{i}^{*}}
$$

The advanced intuitionistic membership function has an additional component to incorporation the indecisiveness.It is defined as,

$$
u_{i k}^{*}=u_{i k}+\pi_{i k} \text {, where } u_{i k}^{*} \text { denotes the }
$$

intuitionistic fuzzy and $u_{i k}$ is the conventional FCM membership function.It denotes the probability of the $\mathrm{k}^{\text {th }}$ data in the $i^{\text {th }}$ class.

$\pi_{i k}$ is hesitation factor, which is given as:

$$
\begin{aligned}
\pi_{i k} & =1-u_{i k}-N\left(u_{i k}\right) \\
\text { and } \pi_{i}^{*} & =\frac{1}{N} \sum_{k=1}^{n} \pi_{i k} \quad \mathrm{k} \in[1, \mathrm{~N}]
\end{aligned}
$$

Intuitionistic fuzzy entropy (IFE) constitues the second term in the objective function.It's addition is done to make sure the maximization the good points in the class and minimizing the entropy of the histogram of an image is the goal.

Cluster centers are modified as,

$$
v_{i}=\frac{\sum_{k=1}^{n} u_{i k}^{*} y_{k}}{\sum_{k=1}^{n} u_{i k}^{*}}
$$

The membership matrix and the cluster center are updated with every iteration.The algorithm terminates when the updated membership and the previous membership agree to the following conditions,

$$
\operatorname{Max} \mid \mathrm{U}_{\mathrm{ik}}{ }^{* \text { new }}-\mathrm{U}_{\mathrm{ik}}{ }^{*} \text { prev } \mid<\beta
$$

$\beta$ is user defined value.

\section{IFCM ALGORITHM WITH NEW INTUITIONISTIC FUZZY COMPLEMENT}

Intuitionistic fuzzy image is constructed from Intuitionistic Fuzzy Generetor(IFG).Non membership values are calculated using intuitionistic fuzzy complement,IFS becomes,

$$
A^{I F S}=\left\{<x, \mu_{A}(x), N\left(\mu_{A}(x)\right)>/ x \in X\right\}
$$

with

hesitation

degree

as,

$\pi_{A}(x)=1-\mu_{A}(x)-N\left(\mu_{A}(x)\right)$

In[3] T.Chaira used Sugeno IFG to construct intuitionistic fuzzy image.Sugeno's intuitionistic fuzzy complement is,

$N(\mu(x))=\frac{1-\mu(x)}{1+\lambda \mu(x)}, \lambda>0, N(1)=0, N(0)=1$

In[5]Deepali Aneja and Tarun Kumar Rawat used Yager IFG to construct intuitionistic fuzzy image.Yager's intuitionistic fuzzy complement is,

$$
N(\mu(x))=\left(1-\mu(x)^{\alpha}\right)^{1 / \alpha}, \alpha>-1
$$

In this study the complement described by George J.Klir and Bo Yuan [6] is used to construct intuitionistic fuzzy image.The intuitionistic fuzzy complement is,

$$
N(\mu(x))=\frac{\gamma^{2}(1-\mu(x))}{\gamma^{2}(1-\mu(x))+\mu(x)}, \gamma>0
$$

\section{RESULTS AND DISCUSSION}

The IFCM algorithm with three different IFG is implemented in MATLABR2009a and performance analysis of the segmentation results are carried out on MR Brain image and Brain tumour MR image.In order to examine the performance and accuracy of segmentation the following assumptions are made

$$
\beta=0.00001, q=2, \alpha=0.85, \lambda=2, \gamma=0.55
$$

and the total number of iterations as 200.The specification for the MR brain image considered for the experimentation is $225 \times 224 \times 3$ pixels

Fig 1(a) represents the original MR brain image and the clustering outcome is shown in Fig 1(b)-(d) using the complement defined by Sugeno,Yager and George J.Klir and Bo Yuan respectively.The specification for the Brain tumour MR image considered for the experimentation is $300 \times 336 \times 3$ pixels.Fig 2(a) represents the original Brain tumour MR image and the clustering outcome is shown in Fig 2(b)-(d) 
using the complement defined by Sugeno, Yager and George J.Klir and Bo Yuan respectively.

After experimenting,proposed method gives the better segmentation results than the existing methods.From Table-1

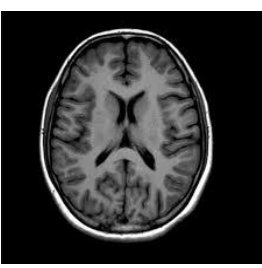

(a)

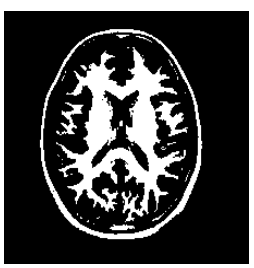

(b) one more result is concluded that number of iteration and execution time is also reduced by proposed method.

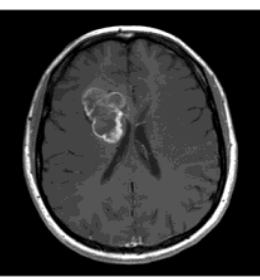

(a)

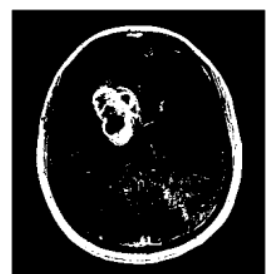

(b)

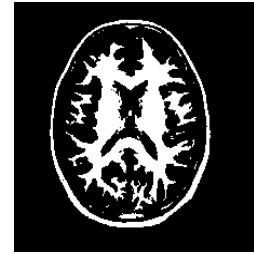

(c)

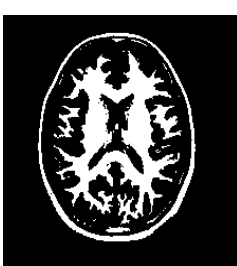

(d)

Fig 1: (a)Original MR brain image (b)Image using IFCM by Chaira (c)Image using IFCM by Deepali Aneja and Tarun Kumar Rawat (d) Image using Proposed IFCM.

Fig 2: (a)Original Brain tumour MR image (b)Image using IFCM by Chaira (c)Image using IFCM by Deepali Aneja and Tarun Kumar Rawat (d) Image using Proposed IFCM.

Table 1. Performance comparison of three methods

\begin{tabular}{|c|c|c|c|c|}
\hline Image & No.of.cluster & Method & No.of.iteration & Duration(secs) \\
\hline MR Brain image & \multirow{2}{*}{4} & Sugeno's complement & 41 & 10 \\
& & Yager's complement & 41 & 8 \\
\hline $\begin{array}{c}\text { Brain tumour MR } \\
\text { image }\end{array}$ & 4 & Proposed & 38 & 7 \\
& & Sugeno's complement & 42 & 11 \\
& & Yager's complement & 39 & 5 \\
\hline
\end{tabular}

\section{CONCLUSION}

This paper provides a new approach for IFCM algorithm by using Intuitionistic fuzzy complement[6].From the experimental results it can be seen that the performance of proposed method has better segmentation according to the segmentation results and the values of converging rate and computational time as compared to the existing methods[3,5] for the considered MR brain image.In future, the proposed method is experimenting on other medical images.

\section{REFERENCES}

[1] Atanassov K.T.1999.Intuitionistic fuzzy sets theory and applications.Physica-Verlag,New York.

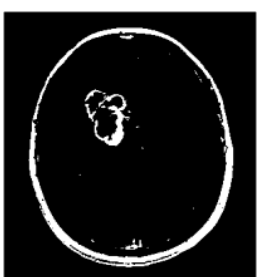

(c)

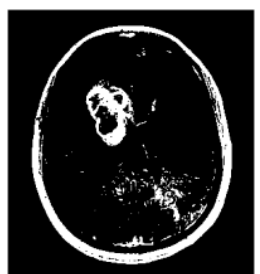

(d)
[2] Bezdek J.C.1981.Pattern recognition with fuzzy objective function algorithms.Plenum,New York.

[3] Chaira T.2011.“'Intuitionistic fuzzy set theory in medical imaging." International Journal of Soft Computing and Engineering,Vol.1,35-37,ISSN:2231-2307.

[4] Chaira T.2011.“A novel intuitionistic fuzzy c- means clustering algorithm and its application to medical images." Applied Soft Computing 11:1711-1717.

[5] Deepali Aneja and Tarun Kumar Rawat.2013."Fuzzy clustering algorithms for effective medical image segmentation." I.J.Intelligent systems and Applications 11:55-61. 
[6] Klir G.J.and Yuan B. 2002.Fuzzy sets and fuzzy logic theory and applications.Prentice Hall of India Private Limited New Delhi.

[7] MR image data set, January, .2012. URL:http://myweb.msoe.edu/ martynsc/images/mri/mri. html.
[8] www.mathworks.com.

[9] Zadeh L.A.1965. "Fuzzy Sets." Information and control 8:338-353. 